\title{
La patologia da Citomegalovirus nel paziente portatore di trapianto renale: impatto, prevenzione e trattamento
}

Giornale di Tecniche Nefrologiche e Dialitiche 2019, Vol. 31(2) 94-99

(C) The Author(s) 2019

Article reuse guidelines:

sagepub.com/journals-permissions

DOI: $10.1177 / 0394936219846415$

journals.sagepub.com/home/gtn

(S)AGE

\author{
Carlo Alfieri',2, Maria Teresa Gandolfo', Evaldo Favi', \\ Marianna Tangredi', Paola Monciino' e Piergiorgio Messa ${ }^{1,2}$
}

\begin{abstract}
Cytomegalovirus infection during renal transplantation

Renal transplantation (RT) is considered the best therapy for patients with chronic renal failure. Renal transplant patients have an elevated risk of infections, and viral diseases have a high prevalence, especially during the first year after RT. Viral infections might influence, in the short and long term, the graft outcome and the patient's survival.

Cytomegalovirus (CMV) disease can be particularly dangerous in immunosuppressed patients and its evaluation is important in the follow-up of RT.

The aim of this review is to present a brief analysis of the epidemiology, clinic characteristics and methods currently available to the clinician for the diagnosis of CMV diseases. The impact, risk factors, main preventive and therapeutic measures currently available to manage CMV diseases in the early stages of RT will also be described, referring to the recent guidelines on the CMV management in transplanted patients.
\end{abstract}

\section{Keywords}

Cytomegalovirus, Prevention, Renal transplantation, Treatment

\section{Introduzione}

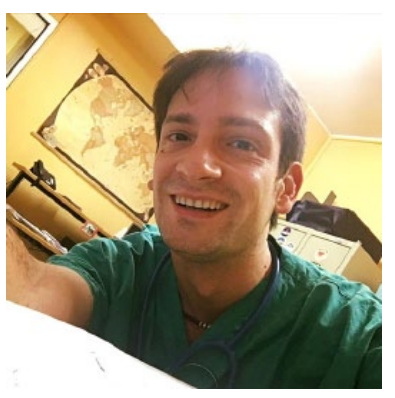

Il trapianto renale (TR) è oggi considerato la migliore terapia per i pazienti affetti da insufficienza renale cronica. I pazienti portatori di TR (TRp) presentano, soprattutto nei primi mesi post-TR, un elevato rischio infettivo legato a fattori clinici pre-TR $\mathrm{e}$ amplificato da fattori più specifici del TR stesso. Le patologie virali, in particolare, hanno una prevalenza piuttosto elevata e possono influire negativamente, a breve e a lungo termine, sulla buona riuscita del TR e sulla sopravvivenza dei TRp.

Il Citomegalovirus (CMV) è un virus ubiquitario che appartiene alla famiglia degli Herpesviridae, e precisamente alla sottofamiglia Betaherpesvirinae. L'infezione o la malattia da CMV, le cui esatte definizioni e differenze verranno spiegate più dettagliatamente nei prossimi paragrafi, sebbene nella popolazione generale siano spesso asintomatiche o paucisintomatiche, possono essere particolarmente pericolose nei pazienti immunosoppressi. Pertanto, il monitoraggio, la prevenzione e il corretto trattamento della patologia da CMV (CMVp) sono molto importanti nel follow-up dei TRp.

\footnotetext{
'U.O. di Nefrologia, Dialisi e Trapianto renale, Fondazione IRCCS Ca Granda, Ospedale Maggiore Policlinico, Milano, Italy

${ }^{2}$ Dipartimento di Scienze Cliniche e di Comunità, Università degli Studi di Milano, Milano, Italy

3U.O. di Trapianto Renale, Fondazione IRCCS Ca Granda, Ospedale Maggiore Policlinico, Milano, Italy

\section{Corrispondenza:}

Dr. Carlo Alfieri, Fondazione IRCCS Ca' Granda, Ospedale Maggiore Policlinico, Milano, Via Commenda I5, 20122 Milan, Italy

E-mail: carlo.alfieril@gmail.com
} 


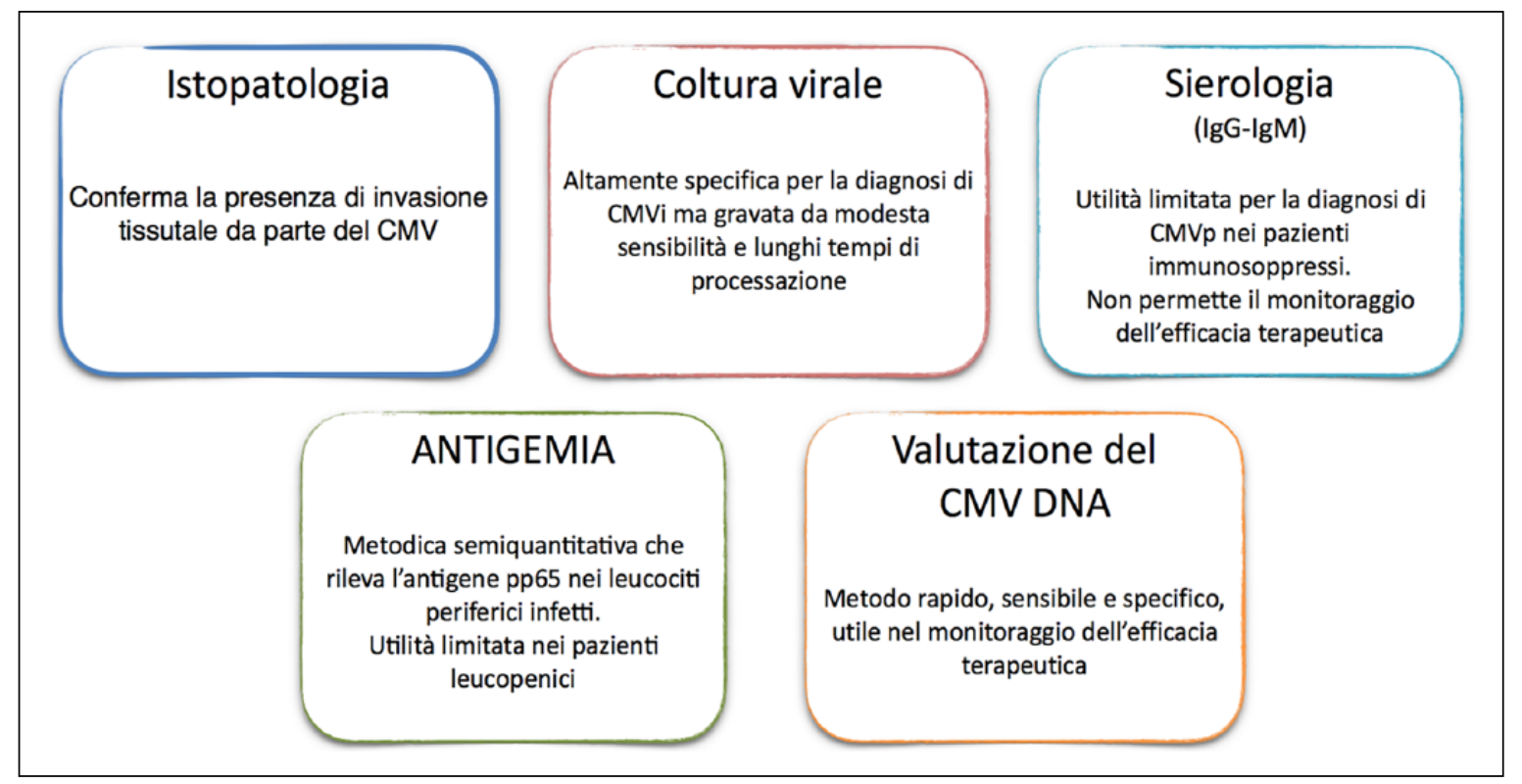

Figura I. Diagnosi laboratoristica di CMVp.

L'obiettivo di questa review è di presentare al lettore una breve analisi dell'epidemiologia, della clinica e dei metodi oggi a disposizione del clinico per la diagnosi del CMVp. Verranno inoltre descritti l'impatto, i fattori di rischio, le principali misure preventive e terapeutiche oggi disponibili per combattere la CMVp nelle prime fasi del $\mathrm{TR}$, facendo riferimento alle recenti linee guida sulla gestione della CMVp nei pazienti trapiantati. ${ }^{1}$

\section{Caratteristiche generali del CMV: epidemiologia, clinica e diagnosi}

Il CMV è un virus molto diffuso nella popolazione mondiale. La sieroprevalenza infatti, si attesta in Europa e negli Stati Uniti attorno al 60\%-70\% della popolazione adulta, mentre nei paesi in via di sviluppo può raggiungere addirittura il $100 \%{ }^{2} \mathrm{Il}$ virus può esser facilmente trasmesso con il contatto tra umani attraverso fluidi corporei, tra cui saliva, urine, secrezioni respiratorie, sangue, feci, e latte materno. ${ }^{3}$

Prima di affrontare le caratteristiche cliniche specifiche della CMVp, è importante sottolineare la differenza tra infezione da CMV e malattia da CMV.

Con il termine infezione da CMV (CMVi), si definisce il riscontro, indipendentemente dalla presenza di sintomatologia clinica, di replicazione ematica del virus.

La malattia da CMV (CMVm) è invece definita dalla presenza di CMVi associata a sintomatologia CMV correlata (febbre, malessere, leucopenia, trombocitopenia, ecc.).

Nei soggetti sieronegativi immunocompetenti, il primo contatto con il virus si presenta frequentemente pauci o asintomatico. In alcuni casi, i pazienti possono presentare una sintomatologia aspecifica, simile ad una prima infezione da Epstein Barr Virus ("CMV mononucleosi"), associata anche a modesto incremento degli enzimi di necrosi epatica. In seguito all'infezione primaria, il CMV stabilisce una fase di infezione non replicante (latenza) localizzandosi nel nucleo delle cellule infettate mieloidi $\mathrm{CD} 34^{+}$in forma episomiale, con possibilità di riattivazione in seguito a stimoli innescanti (prevalentemente immunitari) e in base alle condizioni di immunocompetenza e di comorbilità del soggetto portatore. ${ }^{4}$

In presenza di stati di immunosoppressione severi, una prima infezione da CMV può essere particolarmente rilevante dal punto di vista clinico, coinvolgendo numerosi organi e apparati. La presentazione clinica più comune è caratterizzata dalla presenza di febbre, artralgie, mialgie e mielosoppressione, associata talora a segni e sintomi più specifici di diversi organi e apparati, come il tratto gastroenterico (colite, piuttosto frequente), l'apparato cardio-polmonare (pericardite/miocardite e polmonite), il sistema nervoso centrale e l'apparato visivo (encefalite/ retinite). In caso di trapianto, l'organo trapiantato può esser direttamente coinvolto acutamente, nel breve termine, e cronicamente, nel lungo termine. ${ }^{5}$

È evidente quindi come nella pratica clinica sia fondamentale poter utilizzare dei test diagnostici quanto più specifici, sensibili e di rapida refertazione, che permettano una rapida identificazione del virus e soprattutto un monitoraggio accurato dell'efficacia dell'eventuale terapia prescritta.

Attualmente la diagnosi laboratoristica si avvale principalmente di cinque metodi (figura 1):

- Istopatologia: la diagnosi istologica permette di confermare con certezza la presenza del virus 
all'interno dell'organo invaso attraverso l'identificazione di modificazioni citopatiche CMV-correlate, o l'analisi della presenza di antigeni del virus mediante tecniche di immunoistochimica. ${ }^{6}$ I principali limiti di questo metodo diagnostico sono rappresentati dall'invasività della procedura di prelievo e dalla relativa lentezza nell'analisi del campione. Questa procedura oggi viene riservata solo a quei casi in cui la CMVm sia in diagnosi differenziale $o$ in concomitanza con altri quadri patologici (ad es. rigetti d'organo).

- Coltura virale: questo metodo, sempre meno utilizzato e disponibile in pochissimi centri, può impiegare diverso tempo (settimane) ed il suo utilizzo si è notevolmente ridotto dopo l'immissione in commercio dei kit di biologia molecolare.

- Sierologia: la valutazione della sierologia IgGIgM per CMV permette di conoscere se il soggetto analizzato è già entrato in contatto con il virus in passato. Questa informazione è importante nella valutazione pre-TR e al momento del TR. La sierologia per CMV deve essere valutata sia nel ricevente che nel donatore, in quanto, come vedremo, la sieronegatività del ricevente associata alla sieropositività del donatore può influire sulla condotta preventiva per CMVp nel post-TR. Le linee guida raccomandano la rivalutazione della sierologia CMV (IgG/IgM) nel ricevente IgG- al momento del TR. ${ }^{1}$ L'analisi sierologica può esser utile anche nella valutazione della sieroconversione in seguito a prima CMVi, anche se frequentemente, a causa dell'immunosoppressione, i TRp possono ritardare o non montare affatto una risposta anticorpale specifica contro $\mathrm{CMV} .^{7}$

- Antigenemia: questo test diagnostico è basato sulla rilevazione immunocitochimica di antigeni precoci (pp65) immediati di CMV nei leucociti del sangue. Questo metodo, altamente sensibile e largamente utilizzato in passato prima dell'avvento delle metodiche di biologia molecolare, presenta come unici limiti la tendenza a sottostimare il grado di infezione virale nei pazienti leucopenici e la necessità di trattamenti particolari del campione sanguigno prima dell'analisi. In passato in ogni caso era un buon metodo, utile anche nel monitoraggio dell'efficacia terapeutica. ${ }^{8}$

- Test di biologia molecolare: è oggi il metodo diagnostico maggiormente utilizzato, grazie alla presenza in commercio di molti kit ad alta sensibilità in grado di quantificare il grado di replicazione virale (CMV DNA/RNA). I test di biologia molecolare, oggi di rapida esecuzione e refertazione, sono fondamentali nel monitoraggio della replicazione durante le fasi di prevenzione e soprattutto nella valutazione dell'efficacia terapeutica in corso di CMVi. ${ }^{9}$
Recentemente in alcuni Centri si è reso disponibile l'esame del QuantiFERON-CMV. Il test permette di misurare i livelli di interferone- $\gamma$ in seguito a una stimolazione in vitro con antigeni del CMV. Nel 2013, Manuel et al. dimostrarono, in uno studio effettuato su 127 pazienti trapiantati, l'utilità del test nel classificare i pazienti a basso, medio o alto rischio di sviluppo di CMVi dopo l'interruzione della terapia profilattica. ${ }^{10}$ Più recentemente, in $30 \mathrm{TRp}$ il test è risultato utile nel determinare il rischio di recidiva di CMVi in pazienti ancora in trattamento antivirale. ${ }^{11}$ Alla luce di questi dati, pur essendo stato recentemente introdotto nella pratica clinica, l'esame del QuantiFERON-CMV rappresenta un utile strumento clinico di monitoraggio e di stratificazione del rischio di infezione e recidiva di CMVp il cui utilizzo sicuramente si implementerà in futuro.

\section{Impatto e fattori di rischio di CMVp nel trapianto renale}

La CMVp ha la massima incidenza a partire dal primo mese e durante il primo anno post-TR. ${ }^{12} \mathrm{E}$ importante ricordare, comunque, che una CMVi può presentarsi in qualsiasi fase del TR, e che è sempre consigliata una sua esclusione in presenza di sospetto clinico.

I soggetti sieronegativi al TR che ricevono un organo da un donatore $\mathrm{CMV}-\mathrm{IgG}^{+}$sono di gran lunga quelli a maggior rischio, in quanto verosimilmente svilupperanno una prima infezione da CMV durante il TR.

Numerosi altri fattori influiscono sul rischio di sviluppare CMVp nel post-TR. Lo stato globale di immunosoppressione del TRp, determinato non sono dallo schema di terapia immunosoppressiva adottato, ma anche da fattori legati al paziente stesso, quali età, stress e comorbilità cliniche, è particolarmente rilevante. Studi clinici hanno ben dimostrato come l'utilizzo di timoglobuline nella terapia di induzione, pratica sempre più frequente e necessaria, così come trattamenti di desensibilizzazione con o senza anticorpi monoclonali, incrementino significativamente il rischio di CMVp nei mesi post-TR. ${ }^{13}$ Altri elementi di cui tener conto sono l'esecuzione di trapianti combinati, o il trapianto di organi marginali con lunghi tempi di ischemia.

Dati presenti in letteratura hanno mostrato come il CMV possa esercitare degli effetti nocivi diretti e indiretti sul TR. In particolare, alcuni lavori hanno documentato che la presenza del virus a livello tissutale del TR influenza in negativo l'outcome stesso del TR a breve e a lungo termine, probabilmente attraverso effetti immunomodulatori. In un lavoro pubblicato da Reishing nel 2006, è stata identificata, su una coorte di 106 TRp, la CMVm (e non la CMVi) come fattore di rischio indipendente per lo sviluppo di rigetto acuto nei 12 mesi post-TR. ${ }^{14}$ Nello stesso anno, con un follow- up di 5 anni, Helanterä el al. dimostrarono che la persistenza del virus nel TR, in biopsie renali eseguite due mesi dopo l'ultima rilevazione di CMV su sangue e/o urine, 
Tabella I. Principali caratteristiche dei farmaci antivirali oggi utilizzati nel trattamento della CMVp.

\begin{tabular}{|c|c|c|}
\hline Farmaco & Meccanismo di azione & Caratteristiche \\
\hline Gancyclovir & $\begin{array}{l}\text { In seguito a fosforilazione a gancyclovir } \\
\text { trifosfato inibizione della sintesi del DNA } \\
\text { virale mediante inibizione competitiva della } \\
\text { DNA-polimerasi + incorporazione nel } \\
\text { DNA virale del virus }\end{array}$ & $\begin{array}{l}\text { - Ottima biodisponibilità ev } \\
\text { - Non raccomandato per la terapia pre-emptive, ma } \\
\text { di prima scelta in presenza di CMVm severa } \\
\text { - Rischio di sviluppo di resistenza se somministrato } \\
\text { per os }\end{array}$ \\
\hline Valgancyclovir & $\begin{array}{l}\text { In seguito ad assorbimento } \\
\text { gastrointestinale, trasformazione in } \\
\text { gancyclovir nelle cellule enteriche e } \\
\text { epatiche. }\end{array}$ & - Facilità di somministrazione per os \\
\hline Valacyclovir & $\begin{array}{l}\text { In seguito ad assorbimento } \\
\text { gastrointestinale, trasformazione in } \\
\text { acyclovir trifosfato nelle cellule enteriche } \\
\text { ed epatiche. Inibizione della sintesi del DNA } \\
\text { virale attraverso inibizione competitiva della } \\
\text { DNA-polimerasi }\end{array}$ & $\begin{array}{l}\text { - Efficace ma gravato da un elevato carico giornaliero } \\
\text { di pillole } \\
\text { - Raccomandato per la terapia pre-emptive } \\
\text { - } \quad \text { Rischio elevato di effetti avversi neurologici } \\
\text { (cefalea/alterazione del sensorio) ed epatobiliari } \\
\text { - Non raccomandato per la terapia pre-emptive }\end{array}$ \\
\hline Foscarnet & $\begin{array}{l}\text { Inibizione selettiva sul sito di legame del } \\
\text { pirofosfato della DNA polimerasi virus- } \\
\text { specifica }\end{array}$ & $\begin{array}{l}\text { - } \quad \text { Non richiede processi di attivazione } \\
\text { - } \quad \text { Utile in presenza di CMV mutante (UL97) } \\
\text { - } \quad \text { Altamente nefrotossico } \\
\text { - } \quad \text { Non raccomandato per la terapia pre-emptive }\end{array}$ \\
\hline Cidofovir & $\begin{array}{l}\text { Pro-farmaco, analogo della citosina. In } \\
\text { seguito ad attivazione enzimatica per } \\
\text { difosforilazione, inibizione della DNA } \\
\text { polimerasi virus-specifica }\end{array}$ & $\begin{array}{l}\text { - } \quad \text { Non richiede processi di attivazione } \\
\text { - } \quad \text { Utile in presenza di CMV mutante (UL97) } \\
\text { - } \quad \text { Altamente nefrotossico } \\
\text { - } \quad \text { Non raccomandato per la terapia pre-emptive }\end{array}$ \\
\hline
\end{tabular}

era strettamente correlata ad una ridotta sopravvivenza del TR a lungo termine. ${ }^{15}$

Questi dati avvalorano l'importanza di corrette strategie di prevenzione e di trattamento della CMVp nel TR.

\section{Strategie di prevenzione e trattamento della CMVp nel paziente trapiantato}

Attualmente gli approcci finalizzati a prevenire la CMVp nel paziente portatore di trapianto renale sono tre: profilassi antivirale, la sorveglianza dopo la profilassi e la terapia pre-emptive. È opportuno specificare che ad oggi nessuno studio clinico randomizzato ha dimostrato la superiorità di un approccio rispetto ad un altro, pertanto è fondamentale adattare la condotta clinica in base alle caratteristiche generali del paziente, del donatore e dell'organo trapiantato.

La profilassi antivirale prevede la somministrazione di farmaci antivirali (AV) a partire da non oltre il decimo giorno post-TR per una durata di 3-6 mesi nei TRp definiti "a rischio". Le linee guida raccomandano questa condotta nei TRp IgG" che ricevono un organo $\mathrm{IgG}+(\mathrm{R}-/ \mathrm{D}+)$, con valutazione del CMV-DNA settimanalmente. ${ }^{1}$ In questi pazienti la profilassi ha l'obiettivo di proteggerli da una quasi certa prima infezione fino almeno al $3^{\circ}-6^{\circ}$ mese post-TR, quindi in condizioni cliniche stabilizzate. Raccomandazioni analoghe sono previste per i TRp nei quali il rischio di CMVp è sensibilmente aumentato: pazienti trattati con timoglobuline, sottoposti a terapie di desensibilizzazione o pazienti sottoposti a TR AB0 incompatibili. ${ }^{1}$ Nella nostra Unità Operativa viene effettuata, da protocollo, la profilassi con AV in tutti questi casi, per una durata di 3 mesi, con monitoraggio 2-3 volte la settimana del CMV-DNA.

Molto importante, soprattutto nei pazienti R-/D+ è la sorveglianza dopo sospensione della profilassi, pratica in ogni caso raccomandabile in tutti $\mathrm{i}$ pazienti in cui la profilassi viene interrotta. Uno degli aspetti positivi della profilassi antivirale è sicuramente l'efficacia protettiva, specialmente nei TR R//D+ nei primi mesi di TR che sono i più critici dal punto di vista clinico e chirurgico. D'altra parte, però, la terapia con antivirali è costosa, ed espone $i$ pazienti ai potenziali effetti tossici dei farmaci (in particolare mielotossicità e nefrotossicità).

La terapia pre-emptive prevede un monitoraggio stretto (da linee guida una volta la settimana almeno per i primi tre mesi post-TR) del CMV-DNA e l'impostazione di terapia antivirale in occasione della prima rilevazione dello stesso, con l'obiettivo di prevenire una CMVm. Limiti di questa strategia sono derivati dalla necessità di frequenti test di biologia molecolare, con associate problematiche logistiche per il paziente.

Attualmente, per la profilassi ed il trattamento della CMVp abbiamo a disposizione almeno cinque diversi farmaci antivirali, le cui principali caratteristiche sono riassunte nella tabella 1 . Per i dosaggi degli AV impiegati nella profilassi e nel trattamento, si fa riferimento alle 
linee guida recentemente pubblicate. ${ }^{1}$ Nella tabella 1 non si farà riferimento alle $\mathrm{CMV}$-immunoglobuline in quanto, in considerazione dei frequenti effetti collaterali legati alla loro somministrazione e dell'avvento dei più nuovi antivirali, il loro uso si è notevolmente ridotto e limitato a trattamenti preventivi in pazienti trapiantati di polmone o piccolo intestino oppure in circostanze particolari. ${ }^{16,17}$

Negli anni passati vi è stato un grosso dibattito su quale farmaco antivirale utilizzare per la prevenzione e il trattamento del CMVp. Premettendo che l'efficacia di tutti i farmaci utilizzabili è stata ben dimostrata in studi randomizzati, recenti studi hanno dimostrato la sostanziale equivalenza in termini di efficacia e tollerabilità tra valgancyclovir e gancyclovir, con aspetti favorevoli in termini di biodisponibilità e carico giornaliero di compresse per il valgancyclovir, che viene quindi utilizzato come primo approccio nella profilassi antivirale. ${ }^{18}$ Essendo tutti questi farmaci potenzialmente nefrotossici, si raccomanda di correggere la posologia del farmaco utilizzato in base al grado di funzione renale, che andrebbe tra l'altro frequentemente monitorata. ${ }^{1}$

Gli stessi AV possono essere utilizzati nel trattamento della CMVm. La durata del trattamento è un argomento piuttosto dibattuto, ed effettivamente in questo le linee guida non ci aiutano particolarmente. Per quello che concerne la nostra esperienza di Centro Trapianti, possiamo dire che probabilmente la durata del trattamento dovrebbe essere individualizzata in base alle caratteristiche del paziente, alla clearance del virus e agli eventuali effetti collaterali della terapia. Ciò che è stato però ben evidenziato in letteratura è che, per abbattere significativamente il rischio di recidiva, la replicazione del virus dovrebbe essere totalmente azzerata prima della sospensione del trattamento. ${ }^{19}$ Nella nostra Unità Operativa, sia in presenza di CMVi che di CMVm, sospendiamo il trattamento con AV dopo almeno 3 negatività di CMV-DNA consecutive.

Sebbene le linee guida non indichino chiaramente una durata minima di trattamento, forniscono alcune importanti raccomandazioni: 1) utilizzare come prima linea, ed in presenza di funzione renale normale valgancyclovir $(900$ mg ogni 12 ore) o gancyclovir ( $5 \mathrm{mg} / \mathrm{kg}$ ogni $12 \mathrm{ore}$, ev); 2) in presenza di una moderata $\mathrm{CMVm}$ è possibile utilizzare il valgancyclovir, mentre in presenza di una CMVm severa è raccomandato l'utilizzo del gancyclovir ev; 3) durante il trattamento, è indispensabile il monitoraggio settimanale del CMV-DNA e della funzione renale con conseguente adeguamento della posologia dei farmaci antivirali utilizzati. ${ }^{1}$

In alcuni casi (circa 1\%-5\%) si può verificare una resistenza ai comuni antivirali (rAV). Lo sviluppo di resistenza è determinato da mutazioni virali a carico dei geni UL97 e/o UL54, responsabili della codifica delle chinasi che effettuano la fosforilazione iniziale del gancyclovir, necessaria all'attivazione del farmaco. I fattori di rischio per lo sviluppo di rAV sono una prolungata esposizione alla terapia antivirale, lo stato $\mathrm{R} / \mathrm{D}+\mathrm{e}$ una terapia immunosoppressiva particolarmente potente. Clinicamente, una persistente o crescente replicazione virale o lo sviluppo di replicazione de-novo in presenza di terapia $\mathrm{AV}$, devono far sospettare la $\mathrm{rAV}$. $\mathrm{Al}$ di là di tutto, comunque, la diagnosi di certezza viene ottenuta mediante metodiche di genetica molecolare, che permettono di valutare sequenze virali modificate direttamente dal sangue, da fluidi corporei o da tessuti. ${ }^{20}$ In presenza di una rAV è necessario valutare la possibilità di una eventuale riduzione o modifica della terapia immunosoppressiva in maniera da limitare quanto possibile l'utilizzo di farmaci come il Foscarnet o il Cidofovir gravati da altissima nefrotossicità. In questo senso si segnalano alcune evidenze riguardo un potenziale effetto protettivo degli inibitori del sistema $\mathrm{m}-\mathrm{TOR}$, necessario al virus per la replicazione, sull'incidenza di CMVi e CMVm. ${ }^{21}$ Questa classe di farmaci immunosoppressori potrebbe quindi essere presa in considerazione non solo nella prevenzione, ma anche nel trattamento della CMVp. Studi prospettici randomizzati e di intervento potranno avvalorare maggiormente questa ipotesi.

\section{Considerazioni finali e prospettive future}

Il principale obiettivo di questa review era di descrivere l'impatto e la gestione (preventiva e terapeutica) della patologia da CMV nel paziente trapiantato di rene. Come si è potuto evincere dai concetti e dai lavori citati nel testo, purtroppo le certezze nella gestione di questa patologia non sono numerose. È fondamentale quindi che la gestione della patologia CMV-mediata nei pazienti trapiantati sia guidata da Centri con adeguata esperienza in ambito trapiantologico e presso i cui laboratori siano disponibili le principali metodiche diagnostiche.

I farmaci oggi disponibili possono aiutarci nel prevenire la replicazione virale nei primi mesi post-TR e nell'evitare quadri morbosi anche piuttosto severi nei pazienti immunosoppressi. Ciononostante, la patologia da CMV resta un elemento fondamentale di cui tenere conto durante tutta la vita del trapianto. Rispetto al passato, grazie a nuovi test diagnostici e di monitoraggio e alla presenza di farmaci antivirali efficaci, la gestione della terapia è decisamente più agevole e sicura.

Per il futuro è lecito comunque attendere la commercializzazione di nuove opzioni terapeutiche, caratterizzate da una maggior potenza, biodisponibilità e soprattutto una minor tossicità renale e sistemica. Molto interessante è anche l'ambito di ricerca sullo sviluppo di vaccini anti-CMV, che potrebbero in futuro risultare molto utili soprattutto nei pazienti con sierologia CMV negativa. ${ }^{22}$ 


\section{Dichiarazione di assenza di conflitto di interessi}

Gli Autori dichiarano di non avere conflitti di interessi.

\section{Finanziamenti}

Gli Autori dichiarano di non aver ricevuto finanziamenti specifici da qualsiasi ente nei settori pubblico, privato o senza fini di lucro.

\section{Bibliografia}

1. Kotton C, Kumar D, Caliendo AM, et al. The Third International Consensus Guidelines on the Management of Cytomegalovirus in Solid-organ Transplantation; Transplantation 2018; 102:900-931.

2. Beam E and Razonable RR. Cytomegalovirus in Solid Organ Transplantation: Epidemiology, Prevention, and Treatment. Curr Infect Dis Rep 2012; 14(6):633-641.

3. Zhang LJ, Hanff P, Rutherford C, Churchill WH and Crumpacker CS. Detection of human cytomegalovirus DNA, RNA, and antibody in normal donor blood. The $J$ Infect Dis 1995; 171(4):1002-1006.

4. Tandon R and Mocarski ES. Viral and host control of cytomegalovirus maturation. Trends Microbiol 2012; 20(8):392-401.

5. Kute VB, Vanikar AV, Shah PR, et al. Post-renal transplant cytomegalovirus infection: study of risk factors. Transplant Proc 2012; 44(3):706-709.

6. Ljungman $\mathrm{P}$, Boeckh $\mathrm{M}$, Hirsch $\mathrm{HH}$, et al. Disease Definitions Working Group of the Cytomegalovirus Drug Development Forum. Definitions of Cytomegalovirus Infection and Disease in Transplant Patients for Use in Clinical Trials. Clin Infect Dis 2017; 64(1): 87-91.

7. Humar A, Mazzulli T, Moussa G, et al. Clinical Utility of Cytomegalovirus (CMV) Serology Testing in High-risk CMV D+/R- Transplant Recipients. Am J Transplant 2005; 5(5):1065-1070.

8. The TH, van der Bij $\mathrm{W}$, van den Berg AP, et al. Cytomegalovirus Antigenemia. Clin Infect Dis 1990; 12(Supplement 7):S737-S744.

9. Razonable RR, Paya CV and Smith TF. Role of the laboratory in diagnosis and management of cytomegalovirus infection in hematopoietic stem cell and solid-organ transplant recipients. J Clin Microbiol 2002; 40(3):746-52.

10. Manuel O, Husain S, Kumar D, et al. Assessment of Cytomegalovirus-Specific Cell-Mediated Immunity for the Prediction of Cytomegalovirus Disease in High-Risk
Solid-Organ Transplant Recipients: A Multicenter Cohort Study. Clin Infect Dis 2013; 56(6):817-824.

11. Kumar D, Mian M, Singer L and Humar A. An Interventional Study Using Cell-Mediated Immunity to Personalize Therapy for Cytomegalovirus Infection After Transplantation. Am J Transplant 2017; 17(9):2468-2473.

12. Karuthu S and Blumberg EA. Common infections in kidney transplant recipients. CJASN 2012; 7(12):2058-2070.

13. Issa NC, Fishman JA and Snydman DR. Infectious Complications of Antilymphocyte Therapies in Solid Organ Transplantation. Clin Infect Dis 2009; 48(6):772-786.

14. Reischig $\mathrm{T}$, Jindra $\mathrm{P}$, Svecová $\mathrm{M}$, et al. The impact of cytomegalovirus disease and asymptomatic infection on acute renal allograft rejection. J Clin Virol 2006; 36(2):146-151.

15. Helanterä I, Koskinen $\mathrm{P}$, Finne $\mathrm{P}$, et al. Persistent cytomegalovirus infection in kidney allografts is associated with inferior graft function and survival. Transplant International 2006; 19(11):893-900.

16. Florescu DF, Abu-Elmagd K, Mercer DF, et al. An international survey of cytomegalovirus prevention and treatment practices in intestinal transplantation. Transplantation 2014; 97(1):78-82.

17. Valantine HA, Luikart H, Doyle R, et al. Impact of cytomegalovirus hyperimmune globulin on outcome after cardiothoracic transplantation: a comparative study of combined prophylaxis with CMV hyperimmune globulin plus ganciclovir versus ganciclovir alone. Transplantation 2001; 72(10):1647-52.

18. Asberg A, Humar A, Rollag H, et al. VICTOR Study Group. Oral valganciclovir is noninferior to intravenous ganciclovir for the treatment of cytomegalovirus disease in solid organ transplant recipients. Am J Transplant 2007; 7(9):2106-2113.

19. Sia IG, Wilson JA, Groettum CM, et al. Cytomegalovirus (CMV) DNA load predicts relapsing CMV infection after solid organ transplantation. J Infect Dis 2000; 181(2):717-720.

20. Sahoo MK, Lefterova MI, Yamamoto F, et al. Detection of Cytomegalovirus Drug Resistance Mutations by Next-Generation Sequencing. J Clin Microbiol 2013; 51(11):3700-3710.

21. Andrassy J, Hoffmann VS, Rentsch M, et al. Is Cytomegalovirus Prophylaxis Dispensable in Patients Receiving an mTOR Inhibitor-Based Immunosuppression? A Systematic Review and Meta-Analysis. Transplantation Journal 2012; 94(12):1208-1217.

22. McVoy MA. Cytomegalovirus Vaccines. Clin Infect Dis 2013; 57(supplement4):S196-S199. 